\title{
Economic value of nonsurgical weight loss in adults with obesity
}

Yuchen Ding, PhD; Xiaozhou Fan, PhD, MS; Christopher M Blanchette, PhD, MBA; B Gabriel Smolarz, MD; Wayne Weng, PhD; and Abhilasha Ramasamy, MSc, MS

\section{What is already known about this subject}

- Obesity is a leading contributor to rising health care costs in the United States.

- Approaches such as diet and exercise, antiobesity medications, and bariatric surgery can produce clinically meaningful weight loss (WL).

- The long-term effects of WL on health care costs, particularly for surgical WL, have been studied.

\section{What this study adds}

- There is uncertainty about the effect of nonsurgical WL on health care costs in the short term (i.e., less than 2 years).

- This study analyzed real-world data to estimate the effect of WL on total all-cause health care costs over 1 year for adults in the United States who had nonsurgical WL and those sustained (for an average of 2 years) nonsurgical WL.

\section{ABSTRACT}

BACKGROUND: Obesity imposes a substantial economic burden on the United States. The short-term value of nonsurgical weight loss (WL) and nonsurgical sustained WL (i.e., WL not resulting from bariatric surgery) is poorly understood.

OBJECTIVES: To assess short-term (1 year) effect of nonsurgical WL and sustained nonsurgical WL (i.e., approximately 2 years) on per-patient-per-month (PPPM) total allcause health care costs among adults with obesity in the United States.

METHODS: In this retrospective cohort study, we analyzed data from the IBM MarketScan Explorys Claims-EMR Data Set from January 1, 2012, through June 30, 2018. Adults aged
18-64 years with a body mass index (BMI) measurement $\geq 30 \mathrm{~kg} / \mathrm{m}^{2}$ on the index date and BMI measurements at 12,24 , and 36 months were classified into weight-gain ( $\geq 3 \%$ ), no-weight-change (within $\pm 3 \%$ ), and WL $(\geq 3 \%-\leq 5 \%,>5 \%-\leq 10 \%$, and $>10 \%-\leq 20 \%$ ) cohorts based on the change from first to second BMI measurements (baseline), and sustained nonsurgical WL based on WL during baseline and $<3 \%$ weight gain from second to third BMI measurement. PPPM all-cause health care costs were calculated for baseline, first year, and second year of follow-up. Generalized linear models were used to examine if PPPM all-cause health care cost change ( $\triangle P P P M)$ from baseline to follow-up differed significantly between nonsurgical WL/sustained WL and no-weightchange cohorts. Analyses were stratified by

\section{Author affiliations}

Yuchen Ding, PhD, Health Economics and Outcomes Research; Xiaozhou Fan, PhD, MS, HEOR Real World Data Analytics; Christopher M Blanchette, PhD, MBA, HEOR Strategy and Evidence Generation; B Gabriel Smolarz, MD, US Medical Affairs; Wayne Weng, PhD, HEOR Real World Data Analytics; and Abhilasha Ramasamy, MSc, MS, HEOR Strategy and Evidence Generation, Novo Nordisk, Plainsboro, NJ.

AUTHOR CORRESPONDENCE: Abhilasha Ramasamy, 609.786.6965, arxx@novonordisk.com

J Manag Care Spec Pharm 2021;27(1):37-50

Copyright $\odot 2021$, Academy of Managed Care Pharmacy. All rights reserved.

index obesity class (class 1: BMI $30-<34.9 \mathrm{~kg} / \mathrm{m}^{2}$, class 2: BMI $35-<39.9 \mathrm{~kg} / \mathrm{m}^{2}$, class 3: BMI $\geq 40 \mathrm{~kg} / \mathrm{m}^{2}$ ). Specific nonsurgical WL treatments used by individuals in the study were not studied.

RESULTS: The sample included 20,488 adults who were grouped as follows: weight-gain cohort (24.8\%), no-weightchange cohort (56.6\%), $\geq 3 \%-\leq 5 \%$ WL cohort $(8.2 \%),>5 \%-\leq 10 \%$ WL cohort $(7.7 \%)$, and $>10 \%-\leq 20 \%$ WL cohort (2.8\%). Compared with the no-weight-change cohort, adjusted mean $\triangle$ PPPM all-cause health care cost from baseline to first year of follow-up was lower in all WL cohorts $(\geq 3 \%-\leq 5 \% \mathrm{WL}$ : $-\$ 57.36,>5 \%-\leq 10 \%$ WL: $-\$ 135.35[P<0.05]$, $>10 \%-\leq 20 \%$ WL: $-\$ 193.54[P<0.05])$. In the second year of follow-up $(n=15,307)$, the cohorts were weight-gain (43.4\%), 
no-weight-change (59.4\%), $\geq 3 \%-\leq 5 \%$ sustained WL (7.3\%), $\geq 5 \%-\leq 10 \%$ sustained WL (6.3\%), and $>10 \%-\leq 20 \%$ sustained WL (1.8\%). Adjusted mean $\triangle P P P M$ all-cause health care cost was lower in all sustained WL groups (-\$26.38, $-\$ 157.41[P<0.05]$, and $-\$ 185.41$ for $\geq 3 \%-\leq 5 \%$, $\geq 5 \%-\leq 10 \%$, and $>10 \%-\leq 20 \% W L$, respectively). Greater nonsurgical WL and sustained nonsurgical WL were generally associated with larger reduction in short-term all-cause health care costs. Results stratified by index obesity class were mixed, due to small samples.

CONCLUSIONS: Substantial all-cause health care cost savings were observed 1 year after nonsurgical WL and after sustained (on average for 2 years) nonsurgical WL in adults with obesity, with greater nonsurgical WL and sustained nonsurgical WL associated with greater cost savings. Comprehensive solutions to chronic weight management, including improved access to antiobesity medications in combination with lifestyle interventions, could be valuable to patients, employers, and payers.

The increasing prevalence of obesity in the United States is a significant public health challenge. An estimated $39.8 \%$ of adults in the United States have obesity, which is clinically diagnosed as having a body mass index (BMI) measurement greater than or equal to $30 \mathrm{~kg} / \mathrm{m}^{2}{ }^{1,2}$ Conservative estimates suggest this statistic will rise to $42 \%$ of all U.S. adults by $2030 .^{3}$

As the most significant contributor to the burden of chronic disease, ${ }^{1}$ obesity is associated with increased blood pressure, greater insulin resistance, elevated low-density lipoprotein cholesterol levels, and high triglyceride levels. ${ }^{1}$ In addition to well-known associated increased rates of type 2 diabetes mellitus (T2DM), hypertension, and dyslipidemia, other risks include end-stage renal disease (ESRD), heart disease, certain cancers, and respiratory diseases. ${ }^{1}$

Consequently, obesity imposes a significant economic burden. An estimated $\$ 480$ billion in direct health care costs and $\$ 1.24$ trillion in indirect costs (i.e., costs due to lost economic productivity) were attributed to obesity in 2016. ${ }^{1}$ For people with obesity, per-patient-per-year health care expenditures are an estimated \$4,217 (adjusted to 2019 U.S. dollars [USD]) greater than in those without obesity. ${ }^{4}$

Weight loss (WL) of 5\%-10\% body weight has been established as clinically meaningful and associated with a reduction in comorbidities such as hypertension, hyperlipidemia, and hyperglycemia, and others. ${ }^{5,6}$ Lifestyle interventions and antiobesity medications (AOMs) are nonsurgical options for obesity that can produce WL outcomes in this clinically meaningful range. Diet and exercise have been associated with WL of 5\%-9\% body weight at 6 months and 3\%-6\% body weight at 48 months in a randomized clinical trial. ${ }^{7}$ AOMs approved by the
U.S. Food and Drug Administration (e.g., liraglutide 3.0 mg, naltrexone/bupropion, orlistat, lorcaserin, phentermine/ topiramate) have shown efficacy of $5 \%-10 \%$ WL in phase 3 clinical trials. ${ }^{8-15}$

The definition of sustained WL varies greatly but is most commonly described as that lasting 1 to 2 years, based on a 2017 literature scoping review. ${ }^{16}$ Real-world costs or health care utilization associated with sustained WL were rarely reported $^{16}$; however, economic models have explored longterm (i.e., from 2 years to lifetime) economic benefits of WL. ${ }^{17,18}$ At the individual level, WL of $5 \%$ or $12 \%$ sustained for 2 years was estimated to result in direct medical savings of $\$ 15,800$ or $\$ 26,400$, respectively, per patient over 15 years. ${ }^{18}$ At the population level, the adoption of available AOMs among eligible adults aged $\geq 25$ years was estimated to reduce total medical spending by $\$ 139.2$ billion (2018 USD). ${ }^{17}$

Although models have explored long-term economic effects, short-term health care cost savings for a defined period (i.e., up to 1 year) as a result of nonsurgical WL or sustained nonsurgical WL with current treatments have not been well described..$^{19}$ Also, while the efficacy and economic benefits of surgical WL have been demonstrated, ${ }^{20-23}$ the economic effect of nonsurgical WL has not been comprehensively investigated.

In this study, we describe the short-term (1-year) effect of nonsurgical WL and sustained nonsurgical WL on per-patient-per-month (PPPM) all-cause health care costs, compared with no weight change $( \pm 3 \%)$ in U.S. adults who have obesity. Objective 1 was to assess the short-term (1-year) effect of nonsurgical WL on PPPM all-cause health care costs compared with no weight change in adults who have obesity and how it differs by index obesity class (class 1: BMI $30-<35 \mathrm{~kg} / \mathrm{m}^{2}$; class 2: BMI $35-<40 \mathrm{~kg} / \mathrm{m}^{2}$; class 3: $\left.\mathrm{BMI} \geq 40 \mathrm{~kg} / \mathrm{m}^{2}\right) \cdot{ }^{24}$ Objective 2 assessed the effect of nonsurgical WL sustained over an average of 2 years on PPPM all-cause health care costs compared with patients who had obesity but no weight change over time, and how it differs by index obesity class.

\section{Methods}

\section{STUDY DESIGN}

This retrospective, longitudinal cohort study used data from January 1, 2012, through June 30, 2018, in the IBM MarketScan Explorys Claims-EMR (electronic medical record) Data Set. The dataset is obtained through linkage between an EMR database (IBM Explorys) and a claims database (MarketScan Commercial and Medicare Supplemental Databases) of patients covered by U.S. employer-sponsored private health insurance. ${ }^{25,26}$ All patient-level data within 


\section{TABLE 1 Attrition Table for Study Population}

\begin{tabular}{|c|c|}
\hline Description & $\mathbf{n}(\%)$ \\
\hline Enrolled in commercial insurance within the identification period from January 1, 2012, to June 30, 2014 & $2,644,909 \quad(100)$ \\
\hline Aged $18-64$ years with $\mathrm{BMI} \geq 30 \mathrm{~kg} / \mathrm{m}^{2}$ in identification period & $353,545(13.4)$ \\
\hline Additional 3 BMI measurements identified around 1,2 , and 3 years after index date & $146,092(0.06)$ \\
\hline Continuous enrollment for commercial insurance from index date to date of the last BMI identified & $56,119(0.02)$ \\
\hline Exclude participants with disease diagnosis related to unintentional WL & $44,628(0.02)$ \\
\hline Exclude participants with capitated insurance & $36,630(0.01)$ \\
\hline Exclude participants who have undergone bariatric surgery & $35,592(0.01)$ \\
\hline Exclude participants with WL or WG $20 \%$ & $34,124(0.01)$ \\
\hline $\begin{array}{l}\text { Exclude participants with } \geq 3 \% \text { WG in the first year of follow-up or weight fluctuation in the second year of follow-up } \\
\text { (objective 1) }^{a}\end{array}$ & $20,488(0.01)$ \\
\hline $\begin{array}{l}\text { Exclude participants with } \geq 3 \% \text { WG in the second year of follow-up or weight fluctuation in the second year or third } \\
\text { year of follow-up (objective } 2)^{a}\end{array}$ & $15,307(0.01)$ \\
\hline
\end{tabular}

the MarketScan Research Databases are deidentified and contain synthetic identifiers to help maintain patient privacy. Given the study's retrospective nature and the use of deidentified data, it was exempt from institutional review board approval.

Adults aged 18-64 years were eligible for inclusion if they had a BMI measurement $\geq 30 \mathrm{~kg} / \mathrm{m}^{2}$ on the first instance (index date) of BMI measurement from January 1, 2012, to June 30, 2014. At least 1 additional BMI measurement was required at 1,2 , and 3 years after the index date, each within \pm 6 months. Continuous enrollment during follow-up was required.

Adults were excluded if they had 1 or more diagnostic and procedural codes for conditions or procedures related to unintentional WL or weight gain (WG) at any time during the study period. These included acute or chronic pancreatitis, ESRD, dialysis or renal replacement therapy, feeding difficulty, cirrhosis, cancer, gestational diabetes, pregnancy, and total pancreatic failure. These diagnostic and procedural codes were used in a previous study assessing the economic outcomes associated with weight change in adults who have T2DM. ${ }^{27}$ Individuals with one or more diagnostic and procedural codes for bariatric surgery at any time during the study period were excluded. Diagnostic and procedural codes related to unintentional weight change and bariatric surgery are available from the authors upon request. Those with WG or WL of $>20 \%$ between consecutive BMI measurements were excluded, as extreme changes in weight may indicate bariatric surgery or inaccurate data. Furthermore, those with weight fluctuations (i.e., WG after initial WL or WL after initial weight maintenance) were excluded. Individuals with capitated insurance at any time during the study period were also excluded because this type of insurance enables health care providers to receive the same PPPM payment regardless of health care resource utilization. Those with missing age and sex data were excluded from the adjusted analyses and descriptive tables. Attrition due to the application of the exclusion criteria to the dataset is shown in Table 1.

The index date was defined as the first instance of BMI measurement $\geq 30 \mathrm{~kg} / \mathrm{m}^{2}$ from January 1 , 2012, to June 30, 2014 (identification period). BMI measurements were identified by using code 39156-5 (Logical Observation Identifiers Names and Codes) in EMRs. If multiple measurements were recorded on the same day for an individual, the highest value was recorded to be conservative in the measurement of weight change. As shown in Appendix A (available in online article), BMI measurements occurring 1,2 , and 3 years following the index date ( \pm 6 months) were used to assess changes in PPPM all-cause health care costs from baseline to year 1 (objective 1) and baseline to year 2 (objective 2). A 1-year span was used to capture BMI measurements during the identification period and each year of follow-up to minimize selection bias due to excluding individuals lacking regularly recorded BMI measurements.

Study cohorts were classified based on the difference between the index and second BMI measurements (approximately 1 year $[ \pm 6$ months $]$ after index BMI measurement). These cohorts were no-weight-change $(<3 \% \mathrm{WG}$ to $<3 \% \mathrm{WL}), \geq 3 \%-\leq 5 \% \mathrm{WL},>5 \%-\leq 10 \% \mathrm{WL}$, and $>10 \%-\leq 20 \%$ WL. For objective 2 , sustained WL was defined 
as WL during the baseline and $<3 \%$ WG from the second to third BMI measurement. To rule out the confounding effect of weight fluctuation on the association between WL and PPPM all-cause health care costs, participants in the WL groups were excluded if they had $\geq 3 \%$ WG following their initial or sustained WL. Likewise, subjects in the no-weightchange group were excluded if they had $\geq 3 \%$ WL following initial or sustained weight maintenance.

\section{STATISTICAL ANALYSIS}

PPPM all-cause health care costs were calculated for the baseline, first year of follow-up, and second year of followup (Appendix A). Baseline was defined as the period from the index date to the second BMI measurement. All-cause visits and services used were reported for each measure, including inpatient visits (number of visits and average length of hospital stay), physician-office visits, and emergency room (ER) visits. The number and proportion of patients with such utilization were calculated. Direct medical costs per patient over the variable length of baseline and follow-up periods were measured as a monthly average (i.e., PPPM) and reported for each measure, including inpatient visits, physicianoffice visits, ER visits, and pharmacy costs.

PPPM all-cause health care costs were compared among WL cohorts, the WG cohort, and the no-weight-change cohort in unadjusted and adjusted analyses. Although the WL, WG, and no-weight-change cohorts were all included in the analysis sample, only the WL cohorts were compared with the no-weight-change cohort in the present study.

Multivariable models were used to examine whether PPPM all-cause health care costs changed from baseline to first year of follow-up in the WL cohorts and whether PPPM all-cause health care costs from baseline to second year of follow-up in sustained WL cohorts differed significantly from the no-weight-change cohort. We fit generalized linear models, as the data and errors were normally distributed in our sample. Covariates included in the final models were age, sex, modified Elixhauser Comorbidity Index score, ${ }^{28}$ dyslipidemia, T2DM, osteoarthritis (knee, hip), gastroesophageal reflux disease, hypertension, and musculoskeletal pain. International Classification of Diseases, Ninth Revision, Clinical Modification (ICD-9-CM) and International Classification of Diseases, Tenth Revision, Clinical Modification (ICD-10-CM) codes for comorbidities included in the Elixhauser Comorbidity Index, and obesity-related comorbidities are available from the authors upon request. Adjusted PPPM all-cause health care costs were further stratified by index obesity class (class 1: BMI $30-<35 \mathrm{~kg} / \mathrm{m}^{2}$; class 2: BMI $35-<40 \mathrm{~kg} / \mathrm{m}^{2}$; class 3 : $\mathrm{BMI} \geq 40 \mathrm{~kg} / \mathrm{m}^{2}$ ) to assess effect modification of baseline BMI measurements.
Costs were adjusted to December 2018 USD using the annual medical care component of the Consumer Price Index. All categorical data were assessed using chi-square tests and continuous measures using analysis of variance, with unadjusted comparisons made across weight-change groups. Assuming a power of 0.80 and alpha of 0.05 , it was determined that a sample size of at least 282 would be required to observe a $\$ 1$ reduction in PPPM costs between the $\geq 3 \%-\leq 5 \%$ WL cohort and the no-weight-change cohort. All statistical analyses were conducted using $\mathrm{R}$ version 4.3.3 (R Foundation for Statistical Computing, Vienna, Austria), assuming a 2-tailed test of significance and alpha level set a priori at 0.05 .

\section{Results}

\section{BASELINE DEMOGRAPHICS AND OBESITY-RELATED COMORBIDITIES}

For objective 1, the analysis sample consisted of 15,416 adults aged 18-64 years with obesity (Table 2). Of these, 11,588 patients (56.6\%) had no weight change and 5,072 patients (24.8\%) had WG. Among patients with WL, 1,683 (8.2\%) lost $\geq 3 \%-\leq 5 \%$ body weight; 1,576 (7.7\%) lost $>5 \%-\leq 10 \%$ body weight; and 569 (2.8\%) lost $>10 \%-\leq 20 \%$ body weight. For objective 1, patients had an average of 1.98 years of followup time, with the cohort contributing a total of $30,523 .{ }^{7}$ person-years. For objective 2, patients had an average of 2.96 years of follow-up time and contributed $30,295 .{ }^{6}$ total person-years.

Study cohorts were similar with respect to mean age and BMI class (i.e., class 2 obesity) at index (Table 2). The $>10 \%-\leq 20 \%$ WL cohort had a higher $(65.9 \%)$ proportion of women at baseline relative to other cohorts. Compared with the no-weight-change cohort, WL cohorts had higher prevalence of T2DM (range: $18.8 \%-26.4 \%$ vs. $18.5 \%$ ), gastroesophageal reflux disease (range: $12.3 \%-14.0 \%$ vs. $11.2 \%$ ), and musculoskeletal pain (range: $36.7 \%-42.9 \%$ vs. $35.5 \%$ ), and lower prevalence of knee and/or hip osteoarthritis (range: $3.7 \%-4.1 \%$ vs. $4.5 \%$ ) at baseline.

For objective 2, 2,352 (61.4\%) patients of all 3,828 (100\%) of those with WL sustained it in the second year (Table 2). Sustained WL was observed in 1,113 (7.3\%) patients with $\geq 3 \%-\leq 5 \%$ WL, 964 (6.3\%) patients with $>5 \%-\leq 10 \%$ WL, and 275 (1.8\%) of patients with $>10 \%-\leq 20 \%$ WL. As in the overall sample for objective 1 , the proportion of women (63.3\%) was higher in the $>10 \%-\leq 20 \%$ sustained WL cohort than in other cohorts. Compared at baseline with the no-weight-change cohort, sustained WL cohorts had a higher prevalence of dyslipidemia (range: 46.1\%$48.6 \%$ vs. $43.2 \%$ ), T2DM (range: $23.3 \%-30.1 \%$ vs. $17.5 \%$ ), 


\section{TABLE 2 Baseline Characteristics and Comorbidities by Weight Change Status for Objective 1 and Objective 2}

\begin{tabular}{|c|c|c|c|c|c|c|}
\hline Variable & Total Sample & WG Cohort & $\begin{array}{l}\text { No-Weight- } \\
\text { Change Cohort }\end{array}$ & $\begin{array}{c}\geq 3 \%-\leq 5 \% \text { WL } \\
\text { Cohort }\end{array}$ & $\begin{array}{l}\geq 5 \%-\leq 10 \% \text { WL } \\
\text { Cohort }\end{array}$ & $\begin{array}{l}\geq 10 \%-\leq 20 \% \text { WL } \\
\quad \text { Cohort }\end{array}$ \\
\hline \multicolumn{7}{|l|}{ Objective 1} \\
\hline Sample size, n (\%) & $20,488(100)$ & $5,072(24.8)$ & $11,588(56.6)$ & $1,683 \quad(8.2)$ & $1,576 \quad(7.7)$ & $569 \quad(2.8)$ \\
\hline Age, years, mean (SD) ${ }^{a}$ & $47.9 \quad(9.9)$ & $46.2(10.5)$ & $48.6 \quad(9.5)$ & $48.7 \quad(9.6)$ & $47.9(10.4)$ & $46.3(11.2)$ \\
\hline Female, $\%^{a}$ & 53.7 & 59.3 & 50.7 & 51.7 & 55.8 & 65.9 \\
\hline Index BMI, mean (SD) ${ }^{a}$ & $35.3 \quad(5.4)$ & $35.1 \quad(5.0)$ & $35.3 \quad(5.3)$ & $35.6 \quad(5.6)$ & $35.9(6.0)$ & $36.5 \quad(6.4)$ \\
\hline $\begin{array}{l}\text { Elixhauser Comorbidity Index score, } \\
\text { mean (SD) }\end{array}$ & $0.9 \quad(1.1)$ & $0.9 \quad(1.2)$ & $0.8 \quad(1.1)$ & $0.9 \quad(1.1)$ & $1.0 \quad(1.2)$ & $1.2(1.5)$ \\
\hline \multicolumn{7}{|l|}{ Obesity-related comorbidities, n (\%) } \\
\hline Dyslipidemiaa $^{a}$ & $8,745(42.7)$ & $1,980(39.0)$ & $5,078(43.8)$ & $777(46.2)$ & $685(43.5)$ & $225(39.5)$ \\
\hline Type 2 diabetes mellitus ${ }^{a}$ & $3,854(18.8)$ & $766(15.1)$ & 2,139 (18.5) & $444(26.4)$ & $398(25.3)$ & $107(18.8)$ \\
\hline Osteoarthritis (knee, hip) & $880 \quad(4.3)$ & $204 \quad(4.0)$ & $527 \quad(4.5)$ & $62(3.7)$ & $65 \quad(4.1)$ & $22 \quad(3.9)$ \\
\hline Gastroesophageal reflux disease ${ }^{a}$ & 2,397 (11.7) & $595(11.7)$ & $1,294(11.2)$ & $236(14.0)$ & $194(12.3)$ & $78(13.7)$ \\
\hline Hypertension $^{a}$ & $9,283(45.3)$ & $2,201(43.4)$ & $5,294(45.7)$ & $841(50.0)$ & $701(44.5)$ & $246(43.2)$ \\
\hline Musculoskeletal pain ${ }^{a}$ & $7,482(36.5)$ & $1,904(37.5)$ & $4,112(35.5)$ & $617(36.7)$ & $605(38.4)$ & $244(42.9)$ \\
\hline \multicolumn{7}{|l|}{ Objective 2} \\
\hline Sample size, n (\%) & $15,307(100)$ & $2,201(43.4)$ & $9,097(59.4)$ & $1,113 \quad(7.3)$ & $964 \quad(6.3)$ & $275(1.8)$ \\
\hline Age, mean (SD) ${ }^{a}$ & $48.1 \quad(9.9)$ & $46.3(10.4)$ & $48.6 \quad(9.5)$ & $49.5 \quad(9.2)$ & $49.0(10.0)$ & $47.6(11.0)$ \\
\hline Index BMI, mean (SD) & $35.3 \quad(5.3)$ & $35.1 \quad(5.0)$ & $35.2 \quad(5.2)$ & $35.6 \quad(5.6)$ & $36.1 \quad(6.1)$ & $36.5 \quad(6.3)$ \\
\hline $\begin{array}{l}\text { Elixhauser Comorbidity Index score, } \\
\text { mean (SD) }\end{array}$ & $0.9 \quad(1.1)$ & $0.9 \quad(1.2)$ & $0.8 \quad(1.1)$ & $0.9 \quad(1.1)$ & 1.0 & $1.4(1.7)$ \\
\hline \multicolumn{7}{|l|}{ Obesity-related comorbidities, n (\%) } \\
\hline Dyslipidemia $^{a}$ & $6,560(42.9)$ & $1,514(39.2)$ & $3,932(43.2)$ & $541(48.6)$ & $444(46.1)$ & $129(46.9)$ \\
\hline Type 2 diabetes mellitus ${ }^{a}$ & $2,824(18.4)$ & $549(14.2)$ & $1,591(17.5)$ & $335(30.1)$ & $285(29.6)$ & $64(23.3)$ \\
\hline Osteoarthritis (knee, hip) & $657 \quad(4.3)$ & $150 \quad(3.9)$ & $407 \quad(4.5)$ & $45 \quad(4.0)$ & $41 \quad(4.3)$ & $14 \quad(5.1)$ \\
\hline Gastroesophageal reflux disease ${ }^{a}$ & $1,763(11.5)$ & $454(11.8)$ & $998(11.0)$ & $156(14.0)$ & $116(12.0)$ & $39(14.2)$ \\
\hline Hypertension $^{a}$ & $6,970(45.5)$ & $1,692(43.9)$ & $4,120(45.3)$ & $575(51.7)$ & $454(47.1)$ & $129(46.9)$ \\
\hline Musculoskeletal pain ${ }^{a}$ & $5,507(36.0)$ & $1,445(37.5)$ & $3,174(34.9)$ & $407(36.6)$ & $361(37.4)$ & $120(43.6)$ \\
\hline
\end{tabular}

gastroesophageal reflux disease (range: $12.0 \%-14.2 \%$ vs. $11.0 \%$ ), hypertension (range: $46.9 \%-51.7 \%$ vs. $45.3 \%$ ), and musculoskeletal pain (range: $36.6 \%-43.6 \%$ vs. $34.9 \%$ ).

\section{UNADJUSTED TOTAL PPPM ALL-CAUSE HEALTH CARE COSTS}

In unadjusted analyses, total PPPM all-cause health care costs were lower during the follow-up periods compared with the baseline for all WL categories. For objective 1, unadjusted PPPM all-cause health care cost reductions ranged from $\$ 63.68$ in the $\geq 3 \%-\leq 5 \%$ WL cohort to $\$ 221.36$ in the $>10 \%-\leq 20 \%$ WL cohort from baseline to first year of follow-up (data not shown). A similar trend was observed in the unadjusted analysis for objective 2, with total PPPM allcause health care cost reductions ranging from $\$ 30.42$ in the $\geq 3 \%-\leq 5 \%$ WL cohort to $\$ 263.01$ in the $>10 \%-\leq 20 \%$ WL from baseline to the second year of follow-up (data not shown).

In both objectives, a small reduction in total unadjusted PPPM all-cause health care costs from the baseline to follow-up periods was observed. To understand why baseline costs appeared to be higher overall, we investigated the number of expensive inpatient procedures and the proportion of patients who received them during baseline and follow-up periods (Appendix B, available in online article). 


\section{FIGURE 1 Adjusted $\triangle$ PPPM Total All-Cause Health Care Cost from Baseline to First Year of Follow-up for Nonsurgical WL Compared with No-Weight-Change Cohorts $(n=20,488)$}

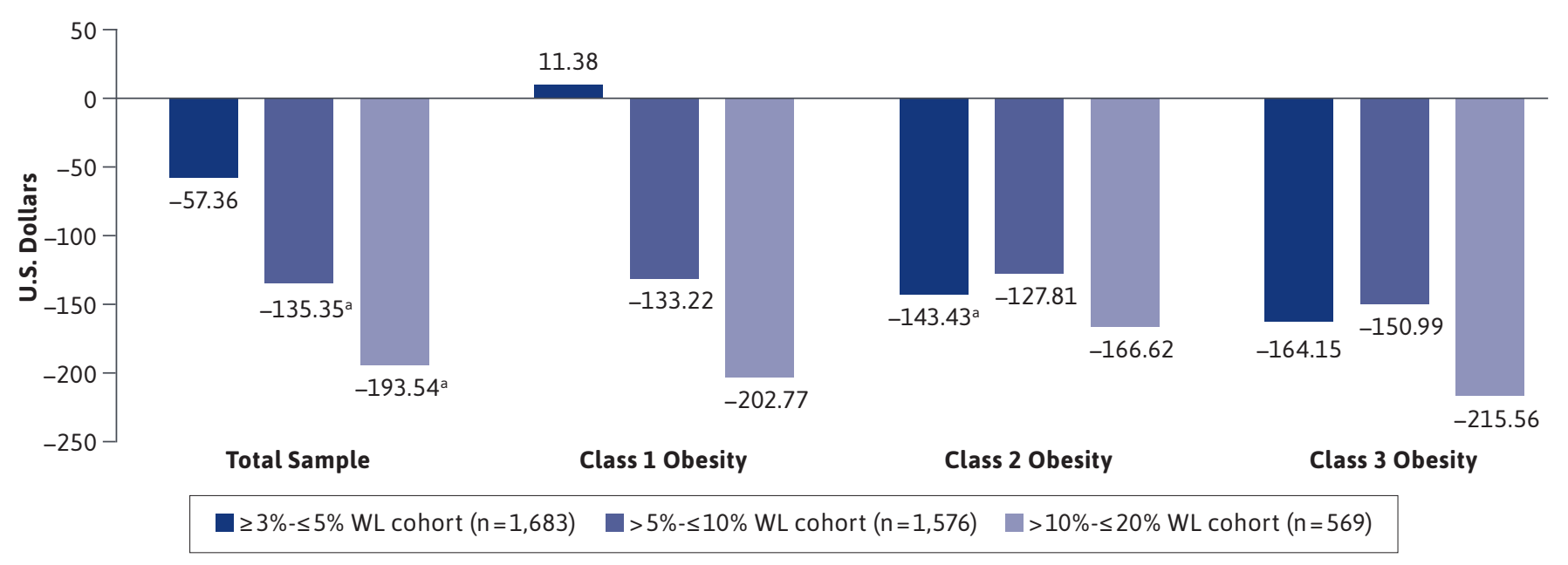

aP $<0.05$.

$\triangle P P P M=$ all-cause health care cost change per patient per month; $W L=$ weight loss.

We noted more inpatient procedures in several major diagnostic categories, including diseases and disorders of the musculoskeletal system and connective tissue, digestive system, and circulatory system, in the baseline than in follow-up periods for objectives 1 and 2. For example, for the first objective, $104(0.7 \%)$ patients received 110 inpatient procedures during baseline that were related to the diagnosis-related group (DRG) code for the musculoskeletal system and connective tissue. This decreased to $71(0.5 \%)$ patients and 78 procedures in the first year of follow-up (Appendix B). This DRG code includes procedures such as major joint replacement or reattachment of lower extremity, spinal fusion except cervical, cervical spinal fusion, back and neck procedure excluding spinal fusion, and revision of hip or knee replacement.

\section{ADJUSTED TOTAL PPPM ALL-CAUSE HEALTH CARE COSTS}

For objective $1(n=20,488)$, adjusted total PPPM all-cause health care cost reductions ranged from \$57.36 PPPM to $\$ 193.54$ PPPM, with the greatest all-cause health care cost reduction observed in the $>10 \%-\leq 20 \%$ WL cohort (Figure 1). These all-cause health care cost differences $(\Delta)$ were statistically significant in the $>5 \%-\leq 10 \%$ WL $(\Delta-\$ 135.35)$ and $>10 \%-\leq 20 \%(\Delta-\$ 193.54)$ WL cohorts. When stratified by index obesity class, the largest PPPM all-cause health care cost reductions were observed in the $>10 \%-\leq 20 \%$ WL cohort (class 1: $\Delta-\$ 202.77$; class 2: $\Delta$-\$166.62; class 3: $\Delta-\$ 215.56)$.

For objective $2(\mathrm{n}=15,307)$, adjusted total PPPM allcause health care cost reductions ranged from $\$ 26.38$ PPPM in the $\geq 3 \%-\leq 5 \%$ sustained nonsurgical WL cohort to $\$ 185.41$ PPPM in the $>10 \%-\leq 20 \%$ sustained nonsurgical WL cohort (Figure 2). As was seen with WL versus no-weight-change cohorts, the largest adjusted PPPM all-cause health care cost reduction occurred in the $>10 \%-\leq 20 \%$ sustained nonsurgical WL cohort. The reduction of $\$ 157.41$ PPPM in the $>5 \%-\leq 10 \%$ sustained nonsurgical WL cohort was statistically significant. In the stratified analysis, the largest adjusted PPPM all-cause health care cost reduction $(\triangle-\$ 248.48)$ was observed in the $>10 \%-\leq 20 \%$ sustained nonsurgical WL cohort in individuals with index obesity class 2 . For the $>5 \%-\leq 10 \%$ sustained nonsurgical WL cohort, the largest adjusted PPPM all-cause health care cost reduction $(\Delta-\$ 211.90 ; \mathrm{P}<0.05)$ was observed in individuals who were in index obesity class 1.

\section{ADJUSTED PPPM RESULTS BY TYPE OF HEALTH CARE COSTS}

Table 3 presents analysis results in the overall sample and stratified by index obesity class. In the overall sample for objective $1(n=20,488)$, statistically significant reductions in inpatient costs were observed for the $>5 \%-\leq 10 \%$ (\$50.34) and $>10 \%-\leq 20 \%$ (\$93.22) WL cohorts (Table 3). Outpatient costs 


\section{FIGURE 2 Adjusted $\triangle$ PPPM Total All-Cause Health Care Cost from Baseline to Second Year of Follow-up for Sustained Nonsurgical WL Compared with No-Weight-Change Cohorts $(n=15,307)$}

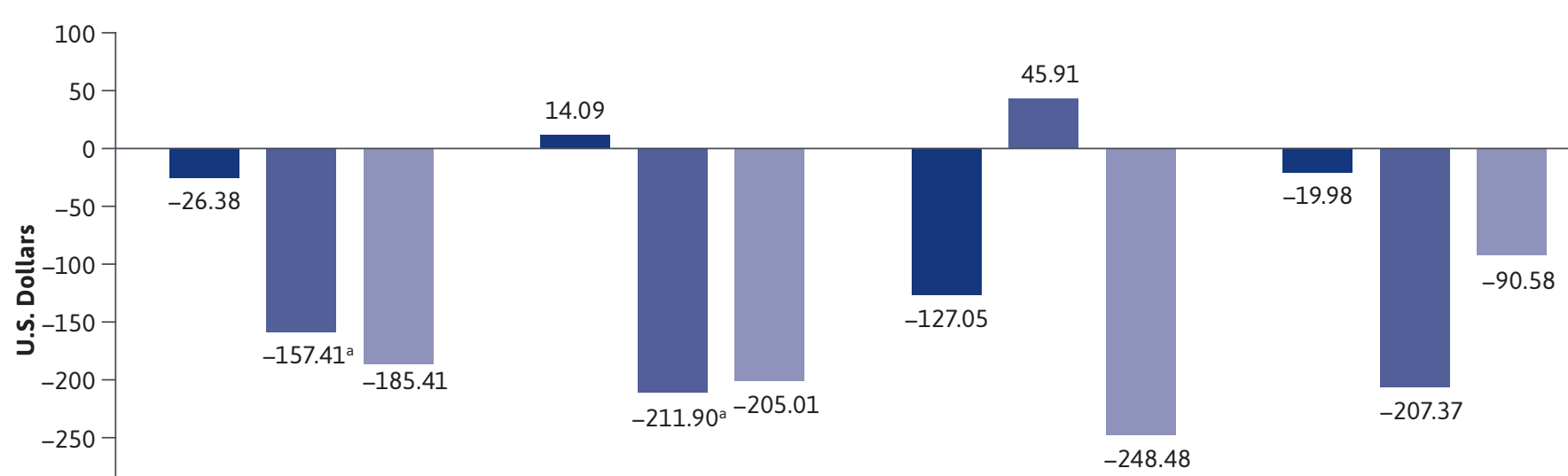

Total Sample

Class 1 Obesity

Class 2 Obesity

Class 3 Obesity

$\geq 3 \%-\leq 5 \%$ sustained WL cohort $(n=1,113)$

$>5 \%-\leq 10 \%$ sustained WL cohort $(n=964)$

$>10 \%-\leq 20 \%$ sustained WL cohort $(n=275)$

${ }^{a} P<0.05$

$\triangle P P P M=$ all-cause health care cost change per patient per month; $W L=$ weight loss.

were also an important contributor, with savings ranging from $\$ 31.33$ PPPM among those with $\geq 3 \%-\leq 5 \%$ WL to $\$ 88.84$ PPPM in those with $>10 \%-\leq 20 \%$ WL. Reductions in ER costs (ranging from $\$ 1.48$ in individuals with $>10 \%-\leq 20 \%$ WL to $\$ 31.41$ in those with $>5 \%-\leq 10 \% \mathrm{WL}$ ) and pharmacy costs ( $\$ 10.00$ in individuals with $>10 \%-\leq 20 \% \mathrm{WL}$ ) were smaller contributors to the reductions in total health care costs. Stratified by index obesity class, results were more mixed, likely due to small samples in the stratified subgroups.

A similar trend was seen for objective $2(n=15,307)$, with statistically significant reductions in inpatient costs for the $>5 \%-\leq 10 \%$ (\$67.63) and >10\%- $\leq 20 \%$ (\$123.22) WL cohorts (Table 3). The greatest outpatient cost savings were in the $>5 \%-\leq 10 \%$ WL cohort $(\$ 97.75[\mathrm{P}<0.05])$; reductions were $\$ 1.87$ in the $\geq 3 \%-\leq 5 \%$ WL cohort and $\$ 46.62$ in the $>10 \%-\leq 20 \%$ WL cohort. There was a small, but statistically significant, reduction $(\$ 1.18[\mathrm{P}<0.05])$ in pharmacy costs for the $\geq 3 \%-\leq 5 \%$ WL cohort.

Results were mixed for the analysis stratified by index obesity class, likely due to small samples. We observed increases in health care costs, rather than cost savings, in some cases; however, most of these results did not reach statistical significance. Among the results that reached statistical significance, most showed that WL was associated with cost reduction.

\section{Discussion}

In this linked EMR-claims database analysis, we found considerable short-term all-cause health care savings 1 year after nonsurgical WL and sustained nonsurgical WL in U.S. adults who had obesity. Overall, greater magnitudes of nonsurgical WL and sustained nonsurgical WL were associated with increased savings. For those achieving $>10 \%-\leq 20 \%$ WL and $>10 \%-\leq 20 \%$ sustained WL, PPPM all-cause health care costs were reduced by $\$ 193.54(\mathrm{P}<0.05)$ from baseline to the first year and by $\$ 185.41$ (not statistically significant) from baseline to the second year, respectively. Although a trend of total health care cost savings was clear in most WL cohorts, the major drivers to cost savings were mainly inpatient and outpatient costs.

While the present observational study could not assess the causal relationship between nonsurgical WL or sustained nonsurgical WL with comorbidity burden, clinical trials have demonstrated that WL can improve outcomes in T2DM and cardiovascular disease. In a meta-analysis of randomized clinical trials of lifestyle interventions, WL of $>5 \%$ was associated with beneficial effects on hemoglobin A1c, lipid, and blood pressure levels in patients with T2DM. ${ }^{29}$ The Look AHEAD randomized clinical trial also demonstrated that WL of $\geq 10 \%$ can reduce the risk of cardiovascular events (a composite of cardiovascular death, nonfatal acute 


\section{TABLE 3 Effect of Weight Loss on Health Care Costs (USD) in Overall Sample and by Index Obesity Class ${ }^{\mathrm{a}}$}

\begin{tabular}{|c|c|c|c|c|}
\hline $\begin{array}{l}\text { Type of } \\
\text { Health Care Cost }\end{array}$ & $\begin{array}{c}\text { No-Weight-Change } \\
\text { Cohort }^{\mathrm{b}}\end{array}$ & $\begin{array}{c}\geq 3 \%-\leq 5 \% \text { WL } \\
\text { Cohort }\end{array}$ & $\begin{array}{c}>5 \%-\leq 10 \% \text { WL } \\
\text { Cohort }\end{array}$ & $\begin{array}{c}>10 \%-\leq 20 \% \text { WL } \\
\text { Cohort }\end{array}$ \\
\hline \multicolumn{5}{|l|}{ Objective 1} \\
\hline \multicolumn{5}{|l|}{ Overall sample } \\
\hline $\mathrm{N}$ & 11,588 & 1,683 & 1,576 & 569 \\
\hline Total costs & Reference & -57.36 & -135.35 & -193.54 \\
\hline Inpatient costs & Reference & -20.45 & -50.34 & -93.22 \\
\hline Outpatient costs & Reference & -31.33 & -65.22 & -88.84 \\
\hline ER costs & Reference & -14.86 & -31.41 & -1.48 \\
\hline Pharmacy costs & Reference & 9.28 & 11.61 & -10.00 \\
\hline \multicolumn{5}{|l|}{ Index obesity class 1} \\
\hline $\mathrm{N}$ & 6,870 & 991 & 909 & 303 \\
\hline Total costs & Reference & 11.38 & -133.22 & -202.77 \\
\hline Inpatient costs & Reference & -19.86 & -61.06 & -45.71 \\
\hline Outpatient costs & Reference & 16.16 & -71.81 & -140.92 \\
\hline ER costs & Reference & -2.34 & -16.44 & -20.46 \\
\hline Pharmacy costs & Reference & 17.43 & 16.08 & 4.32 \\
\hline \multicolumn{5}{|l|}{ Index obesity class 2} \\
\hline $\mathrm{N}$ & 2,855 & 376 & 353 & 137 \\
\hline Total costs & Reference & -143.43 & -127.81 & -166.62 \\
\hline Inpatient costs & Reference & 3.54 & -24.12 & -86.34 \\
\hline Outpatient costs & Reference & -125.25 & -14.91 & -56.70 \\
\hline ER costs & Reference & -35.14 & -87.67 & 11.29 \\
\hline Pharmacy costs & Reference & 13.41 & -1.11 & -34.87 \\
\hline \multicolumn{5}{|l|}{ Index obesity class 3} \\
\hline $\mathrm{N}$ & 1,863 & 316 & 314 & 129 \\
\hline Total costs & Reference & -164.15 & -150.99 & -215.56 \\
\hline Inpatient costs & Reference & -55.71 & -50.98 & -210.05 \\
\hline Outpatient costs & Reference & -60.58 & -94.65 & 3.82 \\
\hline ER costs & Reference & -29.78 & -17.25 & 9.46 \\
\hline Pharmacy costs & Reference & -18.08 & 11.89 & -18.79 \\
\hline \multicolumn{5}{|l|}{ Objective 2} \\
\hline \multicolumn{5}{|l|}{ Overall sample } \\
\hline $\mathrm{N}$ & 9,097 & 1,113 & 964 & 275 \\
\hline Total costs & Reference & -26.38 & -157.41 & -185.41 \\
\hline Inpatient costs & Reference & 3.39 & -67.63 & -123.22 \\
\hline Outpatient costs & Reference & -1.87 & -97.75 & -46.62 \\
\hline ER costs & Reference & -26.73 & -18.95 & -39.57 \\
\hline Pharmacy costs & Reference & -1.18 & 26.91 & 24.01 \\
\hline
\end{tabular}

continued on next page myocardial infarction, nonfatal stroke, and hospital admission for angina) in patients with T2DM. ${ }^{30}$ It is plausible that some health care cost reductions that were observed may be attributable to alleviated comorbidity burden among adults who had WL or sustained nonsurgical WL. Furthermore, our study focused on direct health care costs associated with obesity, but indirect costs account for approximately two thirds of the total costs associated with obesity. ${ }^{1}$ Thus, the total cost savings of WL, from a societal perspective, are potentially greater than our estimates.

Although our study did not assess the economic effect of specific WL treatments, health care providers may consider a holistic treatment approach to obesity. This approach may cover a full spectrum of WL strategies, including lifestyle interventions and AOMs, and bariatric surgery. A recent report by the U.S. Government Accountability Office found that fewer than 1 million adults per year of more than 71 million adults with obesity used an AOM from 2012 to 2016. The report also noted that health insurance coverage was variable and often dependent on prior authorizations or first trying other treatments, such as behavioral modification. ${ }^{31}$

Our results are comparable with prior studies that examined the association between WL and health care cost savings. ${ }^{18,32}$ Using a two-part model of medical expenditures with survey data from noninstitutionalized individuals in the United States, Cawley and Meyerhoefer (2012) examined the relationship between WL and health care costs over 2 years. ${ }^{32}$ Findings indicated substantial decreases in total annual health care costs associated with WL. For instance, health care cost reductions ranged from $\$ 69$ for an index BMI measurement of 30 to $\$ 10,031$ for an index BMI 


\section{TABLE 3 Effect of Weight Loss on Health Care Costs (USD) in Overall Sample and by Index Obesity Class ${ }^{\mathrm{a}}$ (continued)}

\begin{tabular}{|c|c|c|c|c|}
\hline $\begin{array}{l}\text { Type of } \\
\text { Health Care Cost }\end{array}$ & $\begin{array}{c}\text { No-Weight-Change } \\
\text { Cohort }^{\mathrm{b}}\end{array}$ & $\begin{array}{c}\geq 3 \%-\leq 5 \% \text { WL } \\
\text { Cohort }\end{array}$ & $\begin{array}{c}>5 \%-\leq 10 \% \text { WL } \\
\text { Cohort }\end{array}$ & $\begin{array}{c}>10 \%-\leq 20 \% \text { WL } \\
\text { Cohort }\end{array}$ \\
\hline \multicolumn{5}{|c|}{ Index obesity class 1} \\
\hline $\mathrm{N}$ & 5,442 & 654 & 546 & 147 \\
\hline Total costs & Reference & 14.09 & -211.90 & -205.01 \\
\hline Inpatient costs & Reference & 11.61 & -100.65 & -53.39 \\
\hline Outpatient costs & Reference & 24.54 & -132.59 & -129.60 \\
\hline ER costs & Reference & -8.91 & 0.11 & -65.58 \\
\hline Pharmacy costs & Reference & -13.15 & 21.23 & 43.56 \\
\hline \multicolumn{5}{|c|}{ Index obesity class 2} \\
\hline $\mathrm{N}$ & 2,224 & 244 & 214 & 69 \\
\hline Total costs & Reference & -127.05 & 45.91 & -248.48 \\
\hline Inpatient costs & Reference & -27.67 & 3.09 & -42.15 \\
\hline Outpatient costs & Reference & -33.30 & 48.32 & -16.91 \\
\hline ER costs & Reference & -86.52 & -46.48 & -107.36 \\
\hline Pharmacy costs & Reference & 20.43 & 40.98 & -82.05 \\
\hline \multicolumn{5}{|c|}{ Index obesity class 3} \\
\hline $\mathrm{N}$ & 1,431 & 215 & 204 & 59 \\
\hline Total costs & Reference & -19.98 & -207.37 & -90.58 \\
\hline Inpatient costs & Reference & 9.84 & -47.91 & -390.51 \\
\hline Outpatient costs & Reference & -35.24 & -150.53 & 139.96 \\
\hline ER costs & Reference & -8.61 & -35.17 & 64.81 \\
\hline Pharmacy costs & Reference & 14.04 & 26.24 & 95.15 \\
\hline \multicolumn{5}{|c|}{ Note: Bold indicates $P$ value $<0.05$; italic indicates $P$ value $<0.1$. } \\
\hline \multicolumn{5}{|c|}{$\begin{array}{l}{ }^{a} \text { Covariates include age, sex, adjusted Elixhauser Comorbidity Index score, dyslipidemia, type } 2 \text { diabetes } \\
\text { mellitus, osteoarthritis, gastroesophageal reflux disease, hypertension, and musculoskeletal pain. } \\
{ }^{b} \text { No-weight-change cohort was the reference category in the generalized linear model. } \\
\text { ER=emergency room; USD =U.S. dollars; WL=weight loss. }\end{array}$} \\
\hline
\end{tabular}

measurement of 45 (2010 values) for individuals with $5 \%$ WL compared with no WL. ${ }^{32}$ The study estimated greater cost savings in those who had class 2 (range: $\$ 528-\$ 1,608$ ) and class 3 obesity (range: $\$ 2,137-\$ 10,031)$ compared with those who had class 1 obesity (range: \$69-\$394); this trend was consistent for WL of $10 \%, 15 \%$, and $20 \% .^{32}$ This is generally consistent with our results for objective 1, which showed a trend of higher all-cause health care cost savings in patients who had class 3 obesity (range: cost savings of \$150.99-\$215.56) compared with class 1 obesity (range: cost increase of $\$ 11.38$ to cost savings of \$202.77) on the index date. A trend of greater health care cost savings for higher index obesity class was also reported in univariate analyses in the economic model by $\mathrm{Su}$ et al. (2018) (class 1: \$2,581; class 2: \$2,912; class 3: \$4,069); however, in multivariable analyses, health care cost savings for obesity classes 2 and 3 were lower by $5 \%-24 \%$ versus those for obesity class $1 .^{18}$
Another claims-EMR analysis in patients with T2DM found reductions in short-term (6-month and 12-month) health care costs associated with WL. Annual all-cause health care costs were approximately $\$ 500, \$ 800$, and $\$ 1,100$ lower among patients with $3 \%$, $5 \%$, and $7 \% \mathrm{WL}$, respectively, compared with the no-weight-change cohort. ${ }^{27}$ These results are generally comparable with the present study if the results are annualized for the $\geq 3 \%-\leq 5 \% \mathrm{WL}(\$ 688.32)$ and $>5 \%-\leq 10 \%$ WL cohorts $(\$ 1,624.20)$. As in the present study, a greater magnitude of WL was associated with a greater reduction in all-cause health care costs. $^{27}$

Our analysis has several strengths. This is the first large-scale observational study to examine short-term economic effect after nonsurgical WL and sustained nonsurgical WL, using real-world data of working-aged adults with obesity in the United States. Our study comprehensively examined total health care costs, including inpatient visits, physician-office visits, ER visits, and pharmacy costs, in a large sample of patients with WL or no weight change. The linked claims-EMR datasets provided rich clinical context for interpreting utilization and health care costs observed in claims data. Lastly, to reduce potential confounding due to weight fluctuations, the analysis excluded individuals who had subsequent weight regain after their initial WL.

\section{LIMITATIONS}

Our study has several limitations. The study population comprised adults who had obesity and sought care at tier 1 medical facilities, which are centers of excellence, offering comprehensive medical, surgical, psychological, nutritional, and other care. Therefore, results may not be generalizable to patients who seek care in ambulatory or primary care settings. 
Patients with more comorbidities may be more likely to receive diagnostic and procedural codes and have more frequent BMI measurements, potentially leading to selection bias in the EMR data. Data on race and ethnicity and duration of obesity prior to index date were not available. WL associated with specific nonsurgical WL treatments was not assessed. This is because dietary, lifestyle, activity, and behavioral modifications are not likely to be captured accurately in EMRs, and AOM usage was low (<0.5\%) among the study population.

Patients with T2DM may experience WG due to changes in energy expenditure as a result of treatment or WL, difficulty with exercise, the psychological toll of multiple failed WL attempts, ${ }^{33}$ or select classes of antihyperglycemia therapy. ${ }^{34}$ These are potential confounders for analyses of weight change; however, T2DM was included as a covariate in the adjusted analysis. We did not exclude patients with T2DM because health care professionals generally address obesity within the context of comorbidities such as T2DM, and excluding them would have significantly reduced the study sample size.

BMI measurement does not quantify the amount or presence of harmful visceral fat in the way that direct quantitative measures (e.g., dual-energy X-ray absorptiometry imaging) can. However, BMI measurement is the most readily available population-based tool and regarded as the standard diagnostic measure of obesity in clinical practice..$^{35}$ The study sample was small when stratifying patients by index class of obesity, especially in people with $10 \%-20 \%$ WL. This is likely because few individuals can sustain WL of that magnitude for over 1 year. $^{7}$

Furthermore, baseline costs were higher compared with first and second years of follow-up in all WL cohorts and the no-weight-change cohort. We observed that the proportion of patients receiving inpatient procedures was generally higher at baseline, perhaps due to the sample consisting largely of those seeking care at tier 1 medical facilities. Also, baseline costs were highest in patients with $>10 \%-\leq 20 \% \mathrm{WL}$, which may have been due to outliers affecting the mean costs in this small sample.

Finally, the comparison of the WG cohort to the no-weight-change cohort requires additional research. We will compare the WG cohort and the no-weight-change cohort in a future study.

\section{Conclusions}

We demonstrated a substantial shortterm economic value of nonsurgical WL and sustained nonsurgical WL in adults with obesity using real-world data in the United States. Greater allcause health care cost savings were associated with greater WL. Improved access to strategies such as lifestyle interventions and AOM use should be considered by employers and payers to ensure the successful management of obesity-related costs.

\section{DISCLOSURES}

This study was sponsored by Novo Nordisk, which also purchased the data. Blanchette is an employee of Novo Nordisk. Smolarz and Ramasamy are employees of Novo Nordisk and hold equity in Novo Nordisk. Ding, Fan, and Weng were employees of Novo Nordisk at the time this study was conducted.

The findings from this study were previously presented at Obesity Week 2019; November 3-7, 2019; Las Vegas, NV.

\section{ACKNOWLEDGMENTS}

The authors acknowledge the medical writing assistance provided by Karen Kurtyka of Oxford PharmaGenesis (Newtown, PA); this assistance was funded by Novo Nordisk.

\section{REFERENCES}

1. Waters H, Graf M. America's obesity crisis: the health and economic costs of excess weight. Milken Institute; October 2018. Accessed October 9, 2020. https:// www.milkeninstitute.org/sites/default/ files/reports-pdf/Mi-Americas-ObesityCrisis-WEB.pdf

2. Hales CM, Carroll MD, Fryar CD, Odgen CL. Prevalence of obesity among adults and youth: United States, 20152016. NCHS Data Brief, No. 288. October 2017. Accessed October 9, 2020. https:// www.cdc.gov/nchs/data/databriefs/ db288.pdf

3. Finkelstein EA, Khavjou OA, Thompson H, et al. Obesity and severe obesity forecasts through 2030. Am J Prev Med. 2012;42(6):563-70.

4. Cawley J, Meyerhoefer C. The medical care costs of obesity: an instrumental variables approach. J Health Econ. 2012;31(1):219-30. 
5. Tuomilehto J, Lindstrom J, Eriksson JG, et al. Prevention of type 2 diabetes mellitus by changes in lifestyle among subjects with impaired glucose tolerance. N Engl J Med. 2001;344(18):1343-50.

6. Knowler WC, Barrett-Connor E, Fowler SE, et al. Reduction in the incidence of type 2 diabetes with lifestyle intervention or metformin. N Engl J Med. Feb 7 2002;346(6):393-403.

7. Fujioka K. Obesity management: quality of life, cost impact, and disease burden. Am J Pharm Benefits. 2011;3(3):183-90.

8. Apovian CM, Aronne L, Rubino D, et al. A randomized, phase 3 trial of naltrexone SR/bupropion SR on weight and obesityrelated risk factors (COR-II). Obesity (Silver Spring). 2013;21(5):935-43.

9. Davidson MH, Hauptman J, DiGirolamo M, et al. Weight control and risk factor reduction in obese subjects treated for 2 years with orlistat: a randomized controlled trial. JAMA. 1999;281(3):235-42.

10. Davies MJ, Bergenstal R, Bode B, et al. Efficacy of liraglutide for weight loss among patients with type 2 diabetes: the SCALE diabetes randomized clinical trial. JAMA. 2015;314(7):687-99.

11. Fidler MC, Sanchez M, Raether B, et al. A one-year randomized trial of lorcaserin for weight loss in obese and overweight adults: the BLOSSOM trial. J Clin Endocrinol Metab. 2011;96(10):3067-77.

12. Gadde KM, Allison DB, Ryan DH, et al. Effects of low-dose, controlled-release, phentermine plus topiramate combination on weight and associated comorbidities in overweight and obese adults (CONQUER): a randomised, placebo-controlled, phase 3 trial. Lancet. 2011;377(9774):1341-52.

13. Greenway FL, Fujioka K, Plodkowski RA, et al. Effect of naltrexone plus bupropion on weight loss in overweight and obese adults (COR-I): a multicentre, randomised, double-blind, placebo-controlled, phase 3 trial. Lancet. 2010;376(9741):595-605.

14. Pi-Sunyer X, Astrup A, Fujioka K, et al. A randomized, controlled trial of $3.0 \mathrm{mg}$ of liraglutide in weight management. $\mathrm{N}$ Engl J Med. 2015;373(1):11-22.
15. Torgerson JS, Hauptman J, Boldrin MN, Sjostrom L. XENical in the prevention of diabetes in obese subjects (XENDOS) study: a randomized study of orlistat as an adjunct to lifestyle changes for the prevention of type 2 diabetes in obese patients. Diabetes Care. 2004;27(1):155-61.

16. Collins JM, Harvey RA, Ganguly R, Murphy ST, Meade P, Smolarz BG. Defining sustained weight loss: a scoping literature review. Poster presented at: AMCP Annual Meeting 2017. March 27-30, 2017; Denver, CO.

17. Kabiri M, Sexton Ward A, Ramasamy A, et al. The societal value of broader access to antiobesity medications. Obesity (Silver Spring). 2020;28(2):429-36.

18. Su W, Chen F, Dall TM, Zvenyach T, Kyle TK, Perreault L. Where can obesity management policy make the largest impact? Evaluating sub-populations through a microsimulation approach. J Med Econ. 2018;21(9):936-43.

19. Robertson C, Archibald D, Avenell A, et al. Systematic reviews of and integrated report on the quantitative, qualitative and economic evidence base for the management of obesity in men. Health Technol Assess. 2014;18(35):v-vi, xxiii-xxix, 1-424.

20. Garvey WT. New tools for weight-loss therapy enable a more robust medical model for obesity treatment: rationale for a complications-centric approach. Endocr Pract. 2013;19(5):864-74.

21. Lopes EC, Heineck I, Athaydes G, Meinhardt NG, Souto KE, Stein AT. Is bariatric surgery effective in reducing comorbidities and drug costs? A systematic review and meta-analysis. Obes Surg. 2015;25(9):1741-49.

22. Picot J, Jones J, Colquitt JL, et al. The clinical effectiveness and cost-effectiveness of bariatric (weight loss) surgery for obesity: a systematic review and economic evaluation. Health Technol Assess. 2009;13(41):1-190, 215-357, III-IV.

23. Sampalis JS, Liberman M, Auger S, Christou NV. The impact of weight reduction surgery on health-care costs in morbidly obese patients. Obes Surg. 2004;14(7):939-47.
24. Centers for Disease Control and Prevention. Defining adult overweight and obesity. Updated 2017. Accessed October 9, 2020. https://www.cdc.gov/obesity/ adult/defining.html

25. Kaelber DC, Foster W, Gilder J, Love TE, Jain AK. Patient characteristics associated with venous thromboembolic events: a cohort study using pooled electronic health record data. J Am Med Inform Assoc. 2012;19(6):965-72.

26. Hansen L. The IBM MarketScan Research Databases for Life Sciences Researchers. 2018. IBM Watson Health. Accessed October 22, 2020. https://www. ibm.com/downloads/cas/0NKLE57Y

27. Karkare S, Fridman M, Dang-Tan T, et al. Effect of weight change on economic outcomes among persons with type 2 diabetes mellitus in the United States: beyond glycemic control. J Manag Care Spec Pharm. 2019;25(6):658-668. doi: 10.18553/jmcp.2019.18321

28. Quan H, Sundararajan V, Halfon P, et al. Coding algorithms for defining comorbidities in ICD-9-CM and ICD-10 administrative data. Med Care. 2005;43(11):1130-39.

29. Franz MJ, Boucher JL, Rutten-Ramos S, VanWormer JJ. Lifestyle weight-loss intervention outcomes in overweight and obese adults with type 2 diabetes: a systematic review and meta-analysis of randomized clinical trials. J Acad Nutr Diet. 2015;115(9):1447-63.

30. Look AHEAD Research Group, Gregg EW, Jakicic JM, et al. Association of the magnitude of weight loss and changes in physical fitness with long-term cardiovascular disease outcomes in overweight or obese people with type 2 diabetes: a post-hoc analysis of the Look AHEAD randomised clinical trial. Lancet Diabetes Endocrinol. 2016;4(11):913-21.

31. United States Government Accountability Office. Obesity drugs: few adults used prescription drugs for weight loss and insurance coverage varied. GAO-19-577. August 2019. Accessed October 9, 2020. https://www.gao.gov/ assets $/ 710 / 700815 . p d f$ 
32. Cawley J, Meyerhoefer C, Biener A, Hammer M, Wintfeld N. Savings in medical expenditures associated with reductions in body mass index among U.S. adults with obesity, by diabetes status. Pharmacoeconomics. 2015;33(7):707-22.
33. Pi-Sunyer FX. Weight loss in type 2 diabetic patients. Diabetes Care. 2005;28(6):1526-27.

34. Apovian CM, Okemah J, O'Neil PM. Body weight considerations in the management of type 2 diabetes. Adv Ther. 2019;36(1):44-58.
35. Carmienke S, Freitag MH, Pischon T, et al. General and abdominal obesity parameters and their combination in relation to mortality: a systematic review and meta-regression analysis. Eur J Clin Nutr. 2013;67(6):573-85. 


\section{APPENDIX A Assessment of the Effect of Nonsurgical Weight Loss on Health Care Costs}
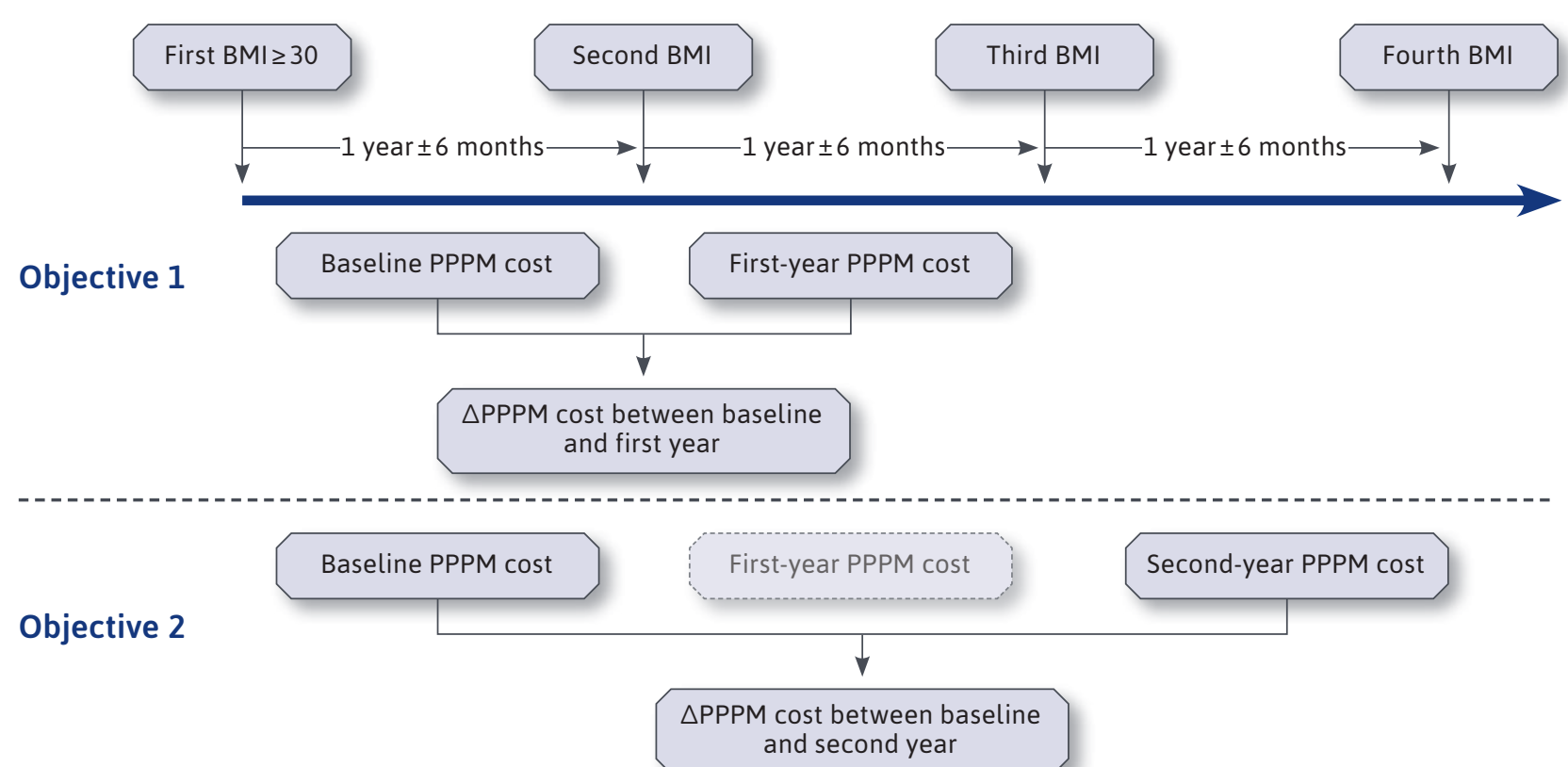

$B M I=$ body mass index; $\triangle P P P M=$ all-cause health care cost change per patient per month. 


\section{APPENDIX B Number of Inpatient Procedures and Average Costs During Baseline and Follow-up Periods}

Major Diagnostic Categories

\begin{tabular}{|c|c|c|c|c|c|c|c|c|}
\hline \multicolumn{3}{|c|}{ Baseline } & \multicolumn{3}{|c|}{ First-Year Follow-up } & \multicolumn{3}{|c|}{ Second-Year Follow-up } \\
\hline$\underline{E}$ & 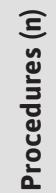 & 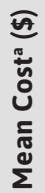 & 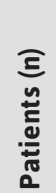 & 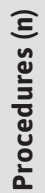 & 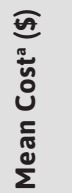 & 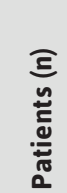 & 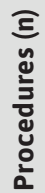 & 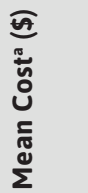 \\
\hline
\end{tabular}

Objective $1(\mathrm{~N}=15,416)$

Diseases \& Disorders of the Musculoskeletal System \& Connective Tissue

Diseases \& Disorders of the Digestive System

Diseases \& Disorders of the Circulatory System

Diseases \& Disorders of the Female Reproductive System

Diseases \& Disorders of the Skin, Subcutaneous Tissue \& Breast

Diseases \& Disorders of the Respiratory System

Factors Influencing Health Status \& Other Contacts with Health Services

Diseases \& Disorders of the Nervous System

Endocrine, Nutritional \& Metabolic Diseases \& Disorders

Diseases \& Disorders of the Kidney \& Urinary Tract

Mental Diseases \& Disorders

Infectious \& Parasitic Diseases, Systemic or Unspecified Sites

Diseases \& Disorders of the Hepatobiliary System \& Pancreas

Injuries, Poisonings \& Toxic Effects of Drugs

Alcohol/Drug Use \& Alcohol/Drug-Induced Organic Mental Disorders

Diseases \& Disorders of the Ear, Nose, Mouth \& Throat

Objective $2(\mathrm{~N}=11,449)$

Diseases \& Disorders of the Musculoskeletal System \& Connective

Tissue

Diseases \& Disorders of the Circulatory System

Diseases \& Disorders of the Digestive System

Diseases \& Disorders of the Female Reproductive System

Diseases \& Disorders of the Respiratory System

Factors Influencing Health Status \& Other Contacts with Health Services

Diseases \& Disorders of the Nervous System

Endocrine, Nutritional \& Metabolic Diseases \& Disorders

Mental Diseases \& Disorders

Diseases \& Disorders of the Skin, Subcutaneous Tissue \& Breast

Diseases \& Disorders of the Kidney \& Urinary Tract

Infectious \& Parasitic Diseases, Systemic or Unspecified Sites

Alcohol/Drug Use \& Alcohol/Drug-Induced Organic Mental Disorders

Diseases \& Disorders of the Hepatobiliary System \& Pancreas

Injuries, Poisonings \& Toxic Effects of Drugs

Diseases \& Disorders of the Ear, Nose, Mouth \& Throat

\begin{tabular}{r|r|r|r|r|r|r|r|c}
\hline 104 & 110 & $32,946.15$ & 71 & 78 & $30,865.72$ & - & - & - \\
\hline 45 & 46 & $19,155.12$ & 23 & 24 & $22,873.53$ & - & - & - \\
\hline 45 & 48 & $37,095.98$ & 28 & 29 & $51,374.47$ & - & - & - \\
\hline 28 & 28 & $14,268.57$ & 15 & 15 & $13,812.90$ & - & - & - \\
\hline 13 & 14 & $8,202.99$ & 7 & 8 & $15,967.63$ & - & - & - \\
\hline 10 & 10 & $36,193.13$ & 10 & 10 & $12,226.65$ & - & - & - \\
\hline 10 & 10 & $12,447.56$ & 6 & 6 & $10,170.44$ & - & - & - \\
\hline 9 & 11 & $21,848.60$ & 7 & 7 & $24,343.36$ & - & - & - \\
\hline 8 & 8 & $19,380.62$ & 2 & 3 & $12,698.93$ & - & - & - \\
\hline 7 & 7 & $13,251.66$ & 3 & 3 & $9,030.41$ & - & - & - \\
\hline 7 & 7 & $6,343.75$ & 7 & 10 & $4,941.96$ & - & - & - \\
\hline 7 & 7 & $15,214.29$ & 3 & 3 & $12,424.11$ & - & - & - \\
\hline 4 & 4 & $19,745.94$ & 2 & 2 & $17,044.14$ & - & - & - \\
\hline 3 & 3 & $64,924.43$ & 4 & 4 & $30,555.70$ & - & - & - \\
\hline 3 & 3 & $5,176.86$ & 4 & 7 & $5,254.05$ & - & - & - \\
\hline 2 & 2 & $19,823.62$ & 4 & 4 & $9,153.77$ & - & - & - \\
\hline
\end{tabular}

\begin{tabular}{|r|r|r|r|r|r|r|r|r}
\hline 73 & 76 & $32,578.31$ & 49 & 55 & $31,206.16$ & 52 & 56 & $37,828.21$ \\
\hline 38 & 41 & $37,452.95$ & 24 & 25 & $45,331.69$ & 21 & 23 & $4,7281.07$ \\
\hline 35 & 36 & $16,851.25$ & 17 & 18 & $24,231.86$ & 12 & 12 & $24,802.92$ \\
\hline 19 & 19 & $15,716.39$ & 9 & 9 & $11,891.30$ & 10 & 10 & $12,577.34$ \\
\hline 9 & 9 & $37,167.51$ & 5 & 5 & $13,835.26$ & 7 & 7 & $14,502.04$ \\
\hline 8 & 8 & $13,150.09$ & 6 & 6 & $10,170.44$ & 0 & 0 & 0.00 \\
\hline 7 & 9 & $14,699.97$ & 7 & 7 & $24,343.36$ & 7 & 9 & $20,534.03$ \\
\hline 6 & 6 & $21,479.93$ & 1 & 1 & $95,46.34$ & 3 & 3 & $42,807.62$ \\
\hline 6 & 6 & $6,496.80$ & 6 & 9 & $48,46.08$ & 6 & 8 & $4,626.75$ \\
\hline 6 & 7 & $9,803.96$ & 5 & 6 & $19,011.07$ & 3 & 3 & $13,723.11$ \\
\hline 5 & 5 & $11,154.56$ & 2 & 2 & $8,652.52$ & 5 & 5 & $25,047.21$ \\
\hline 4 & 4 & $14,881.85$ & 3 & 3 & $12,424.11$ & 2 & 3 & $34,927.55$ \\
\hline 3 & 3 & $5,176.86$ & 3 & 3 & $6,181.81$ & 4 & 7 & $5,845.70$ \\
\hline 3 & 3 & $14,480.01$ & 1 & 1 & $15,724.03$ & 1 & 1 & $17,882.36$ \\
\hline 2 & 2 & $90,621.29$ & 3 & 3 & $39,668.20$ & 0 & 0 & 0.00 \\
\hline 2 & 2 & $19,823.62$ & 2 & 2 & $6,020.46$ & 1 & 1 & $8,364.48$ \\
\hline
\end{tabular}

a Mean cost per patient represented in U.S. dollars per major diagnostic code category. 